Published online 2017 April 13.

Abstract

\title{
Imaging Presentation of Budd-Chiari Syndrome
}

\author{
Atousa Adibi, ${ }^{1, *}$ \\ ${ }^{1}$ Professor of Radiology, Isfahan University of Medical Sciences, Isfahan, Iran \\ "Corresponding author: Atousa Adibi, MD, Professor of Radiology, Isfahan University of Medical Sciences, Isfahan, Iran. E-mail: a_adibi@med.mui.ac.ir
}

Received 2016 December 21; Accepted 2017 February 08.

\begin{abstract}
Budd-Chiari syndrome is caused by the obstruction of the hepatic venous, with high mortality rate in nontreated cases. Because the clinical presentation of this syndrome is nonspecific, is very important. Contrast enhanced multiphase computed tomography (CT) and magnetic resonance (MR) angiography permits morphologic and functional assessment of liver changes in suspicious clinical setting. The aim of this presentation is to present the classical imaging features of this syndrome. 1- Radiographic features, 1-1- CT scan, 1-1-1- inhomogeneous mottled liver with delayed enhancement in the periphery of the liver and around the hepatic veins; 1-1-2peripheral zones of the liver may appear hypo attenuating because of reversed portal venous blood flow; 1-1-3- caudate lobe enlargement and increased contrast enhancement compared with the remainder of the liver; 1-1-4-inability to identify hepatic veins. 1-2-Ultrasound, 1-2-1- acute; 1-2-2- hepatomegaly; 1-2-3- splenomegaly; 1-2-4- heterogeneous echotexture; 1-2-5- chronic; 1-2-6- hypertrophied caudate lobe; 1-2-7- peripheral atrophy; 1-2-8- regenerative nodules; 1-2-9- gallbladder wall thickening; 1-2-10- ascites. 1-3- Color-flow Doppler, 1-3-1- part of or the entire right hepatic vein with no flow or inappropriately directed flow; 1-3-2- discontinuity between the main hepatic vein and the IVC; 1-3-3- reversed flow in hepatic veins and intra and extrahepatic collaterals; 1-3-4- portal vein changes such as hepatopetal or hepatofugal flow; 1-3-5- thrombus or tumour within the IVC;1-3-6-increased resistive index within the hepatic artery: $>0.75$.
\end{abstract}

This is an abstract presented in the 33rd Iranian congress of radiology (ICR) and the 15th congress of Iranian radiographic science association (IRSA). 